\title{
Short-term outcomes of patients with neovascular exudative AMD: the effect of COVID-19 pandemic
}

\author{
Enrico Borrelli ${ }^{1}$. Domenico Grosso ${ }^{1} \cdot$ Giovanna Vella $^{1,2} \cdot$ Riccardo Sacconi $^{1} \cdot$ Marco Battista $^{1} \cdot$ Lea Querques $^{1}$. \\ Ilaria Zucchiatti ${ }^{1}$. Francesco Prascina ${ }^{1} \cdot$ Francesco Bandello $^{1} \cdot$ Giuseppe Querques $^{1}$ (i)
}

Received: 24 August 2020 / Revised: 16 September 2020 / Accepted: 25 September 2020 / Published online: 3 October 2020

(C) Springer-Verlag GmbH Germany, part of Springer Nature 2020

\begin{abstract}
Purpose To estimate the impact of delayed care during the coronavirus disease 2019 (COVID-19) pandemic on the outcomes of patients with neovascular age-related macular degeneration (AMD).

Methods Consecutive patients with diagnosis of neovascular AMD were consecutively enrolled between March 9, 2020, and June 12, 2020, (during and immediately after the Italian COVID-19 quarantine). During the inclusion (or pandemic) visit ( $\mathrm{V}_{0}$ ), patients received a complete ophthalmologic evaluation, including optical coherence tomography (OCT). Best-corrected visual acuity (BCVA) and OCT findings from the two preceding visits $\left(\mathrm{V}_{-1}\right.$ and $\left.\mathrm{V}_{-2}\right)$ were compared with data at $\mathrm{V}_{0}$.

Results One-hundred patients (112 eyes) were enrolled in this study. The time interval between following visits was $110.7 \pm$ 37.5 days within $\mathrm{V}_{0}$ and $\mathrm{V}_{-1}$ and $80.8 \pm 39.7$ days within $\mathrm{V}_{-1}$ and $\mathrm{V}_{-2}$, respectively $(P<0.0001)$. BCVA was statistically worse at the $\mathrm{V}_{0}$ visit as compared with the immediately preceding $\left(\mathrm{V}_{-1}\right)$ visit $(0.50 \pm 0.43 \operatorname{LogMAR}$ and $0.45 \pm 0.38$ LogMAR at the $\mathrm{V}_{0}$ and $\mathrm{V}_{-1}$ visits, respectively; $\left.P=0.046\right)$. On structural OCT, 91 out of $112(81.2 \%)$ neovascular AMD eyes displayed the evidence of exudative disease activity at the $\mathrm{V}_{0}$ visit, while $77(68.7 \%)$ eyes exhibited signs of exudation at the $\mathrm{V}_{-1}$ visit $(P=$ 0.022). No differences in terms of BCVA and OCT findings were detected between the $\mathrm{V}_{-1}$ and $\mathrm{V}_{-2}$ visits. In multiple regression analysis, the difference in BCVA between $V_{0}$ and $V_{-1}$ visits was significantly associated with the interval time within these two visits $(P=0.026)$.

Conclusion The COVID-19 pandemic-related postponement in patient care proved to be significantly associated with worse short-term outcomes in these patients.
\end{abstract}

Keywords COVID-19 · Retina · Neovascular AMD · Outcome

\section{Introduction}

Age-related macular degeneration (AMD) is the leading cause of severe decrease in visual acuity among subjects older than 50 years [1]. Severe vision loss in AMD is frequently the result of development of macular neovascularization (MNV) $[2,3]$. Exudation from MNV may indeed irreversibly damage

This is part of a topical collection on Perspectives on COVID-19

Giuseppe Querques

giuseppe.querques@unisr.it

1 Department of Ophthalmology, University Vita-Salute, IRCCS Ospedale San Raffaele, Via Olgettina, 60 Milan, Italy

2 Ophthalmology, Department of Surgical, Medical, Molecular Pathology and of Critical Area, University of Pisa, Pisa, Italy photoreceptors and retinal pigment epithelium (RPE) in neovascular AMD [4].

Treatment of exudative neovascular AMD has been significantly evolved over the past two decades and anti-vascular endothelial growth factor (VEGF) therapy is currently considered as the gold standard of treatment in these eyes. This treatment proved to be effective for preventing disease progression and improving vision in patients with neovascular AMD [5-7]. However, anti-VEGF therapy must be given at appropriate intervals in order to control disease activity [5-7].

Current treatment strategies include monthly (treatment every 4 weeks) and bimonthly (treatment every 8 weeks after 3 month loading) fixed-interval regimens that were employed in the registration trials for ranibizumab and aflibercept, respectively [8-12]. Although these fixed-interval regimens represent on-label standards for anti-VEGF therapy, discontinuous regimens are mostly used in clinical practice in order to reduce 


\section{Key messages}

- The volume of outpatient visits and intravitreal injections has declined during the COVID-19 pandemic.

- The follow-up of patients with neovascular AMD was significantly postponed during this period.

- Postponement in in-person visits has significantly impacted on the visual acuity and anatomical findings in patients with neovascular AMD.

treatment burden. The "pro-re-nata" (PRN - "as needed") protocol is based on re-treatment on evidence of exudative disease activity [13]. Conversely, the "treat and extend" (TREX) regimen is characterized by a progressive extension of treatment and visit intervals up to 12 weeks depending on the clinical findings [14]. These discontinuous regimens are widely used worldwide as they have been validated in previous studies [15-18]. Their introduction was mainly intended to reducing the number of intravitreal injections as compared with fixed regimens. Importantly, all the above-mentioned regimens include an ocular examination prior to anti-VEGF injection. While in the fixed-interval protocol this assessment is mainly aimed at excluding complications and/or ocular inflammation before therapy, PRN and TREX protocols require a detailed ocular examination in order to adopt a final therapeutic decision.

The coronavirus disease 2019 (COVID-19) pandemic has drastically modified how outpatient care is given in healthcare practices. This disease has hit several regions worldwide and single countries' outbreaks have evolved at different speeds and at distinct times. During the worst phases of COVID-19 outbreak, in order to reduce the chance of transmitting the virus to either patients or healthcare personnel, providers have deferred elective and preventive visits, and outpatient visits have been restricted to more urgent care [19-23]. Symmetrically, many patients have also postponed visits in order to avoid being exposed. A recent report from our group has demonstrated that the COVID-19 pandemic has significantly shrunk both the in-person visit and intravitreal procedure volumes during the COVID-19 quarantine [24]. Importantly, the latter study showed that outpatient care in AMD patients was the most impacted by the COVID-19 pandemic.

Assuming that the number of injections and a failure to visit clinicians and undergo optical coherence tomography (OCT) were demonstrated to be associated with worse outcomes in patients with neovascular AMD [25], the COVID19-related contraction in outpatient volume might result in significant repercussions on the outcome of these patients.

Assuming that there is a lack of informative ophthalmology non-communicable diseases (NCD)-related epidemiology in emergency settings, the aim of this study was to estimate the impact of delayed care secondary to COVID-19 in patients with exudative neovascular AMD. We also examined the relationship between functional and anatomic outcomes and patients' characteristics. It is hoped that these analyses will provide a valuable insight into factors affecting outcomes in reallife emergency settings. More importantly, our findings might be helpful in the management of neovascular AMD patients in the course of second waves of the COVID-19 outbreak.

\section{Methods}

This was a consecutive observational case series that adhered to the 1964 Helsinki declaration and its later amendments. Informed consent was obtained from all individual participants included in the study. The study was approved by the Institutional Review Board (IRB) of the San Raffaele Scientific Institute.

\section{Study participants}

Patients 50 years of age and older with a clinical diagnosis of neovascular AMD in at least one eye were enrolled from a medical retinal practice (Medical Retina and Imaging Unit) at the San Raffaele Scientific institute in Milan, Italy. All patients were consecutively enrolled between March 9, 2020, and June 12, 2020, (during and immediately after the Italian COVID-19 quarantine). During the inclusion (or pandemic) visit $\left(\mathrm{V}_{0}\right)$, patients received a complete ophthalmologic examination, which included the measurement of best-corrected visual acuity (BCVA), dilated ophthalmoscopy, and structural OCT. BCVA measurements were made using a Snellen chart and were converted to the logarithm of the minimum angle of resolution (LogMAR), as previously described [26]. Structural OCT imaging was performed with the Heidelberg Spectralis HRA+OCT device (Heidelberg Engineering, Heidelberg, Germany). Each set of SD-OCT scans consisted of 19 B-scans, each of which comprised 24 averaged scans, covering approximately $5.5 \times 4.5-\mathrm{mm}$ area centred on the fovea. A minimum signal strength of 25 was required to the OCT images to be included, as recommended by the manufacturer [27]. 
The inclusion criteria for enrolled eyes included evidence of exudative neovascular AMD by history or examination and positive history for anti-VEGF therapy with a PRN approach. Furthermore, patients were required to have records of at least two complete ophthalmology examinations $\left(\mathrm{V}_{-1}\right.$ and $\left.\mathrm{V}_{-2}\right)$ prior to the inclusion visit $\left(\mathrm{V}_{0}\right)$. Importantly, $\mathrm{V}_{-1}$ and $\mathrm{V}_{-2}$ were required to be subsequent to the anti-VEGF loading phase, in order to have all the examinations $\left(\mathrm{V}_{0}, \mathrm{~V}_{-1}\right.$ and $\mathrm{V}_{-2}$ ) subsequent to at least 3 anti-VEGF injections. Exclusion criteria for neovascular AMD eyes were (i) any maculopathy secondary to causes other than AMD (including presence of vitreomacular traction syndrome or an epiretinal membrane) and (ii) presence of foveal macular atrophy, as assessed with structural OCT, as this may reduce the capability to discern changes in visual acuity because of worsening in neovascular AMD clinical characteristics [28].

\section{OCT grading}

At the $\mathrm{V}_{0}, \mathrm{~V}_{-1}$ and $\mathrm{V}_{-2}$ visits, OCT images were graded for qualitative features previously proposed as suggestive of exudative disease activity [29-31], including presence of subretinal fluid, intraretinal fluid, and subretinal hyperreflective material (SHRM). Structural OCT images were reviewed by two independent and experienced readers. Based on previous studies [32], a lesion was graded as present if the reader had a more than $90 \%$ confidence that it was recognizable in at least $1 \mathrm{~B}$-scan. Graders later met to compare level of agreement, and disagreements were resolved by open adjudication to yield a single assessment for each case. In those cases in which the graders did not agree on a single consensus result, the final decision was made by the director of the San Raffaele Medical Retina and Imaging Unit (GQ).

\section{Statistical analysis}

Statistical calculations were performed using Statistical Package for Social Sciences (version 20.0, SPSS Inc., Chicago, IL, USA).

Continuous variables were compared by conducting a paired sample $t$ test. Statistical significance of the differences for qualitative variables was assessed using Fisher's exact test.

Relationships between change in BCVA between $\mathrm{V}_{0}$ and $\mathrm{V}_{-1}$ visits (dependent variable) and other clinical features and demographics (independent variables) were evaluated using a multiple regression analysis. In the regression analysis, only the right eyes of patients with bilateral neovascular AMD were included. A $P$ value $<0.05$ was determined to be statistically significant.

\section{Results}

\section{Characteristics of patients included in the analysis}

Clinical characteristics of enrolled patients are presented in Table 1 . We included 100 consecutive patients with neovascular AMD, after excluding 7 patients because they were not fulfilling exclusion criteria. In our study cohort, 49 were female $(49.0 \%)$ and 51 were male $(51.0 \%)$. Mean \pm SD age was $79.1 \pm 7.6$ years (range $60-95$ years). In the study group, 12 patients presented with bilateral neovascular AMD, whereas 88 patients presented with either dry AMD or non-includable neovascular AMD in the fellow eye. Therefore, 112 eyes from 100 patients met the inclusion criteria and were finally included in our analysis. Mean \pm SD previous anti-VEGF injections before inclusion in the study was $15.0 \pm 10.1$ (range 3-46 injections). The number of anti-VEGF injections in the 12 months preceding $\mathrm{V}_{0}$ was $3.3 \pm 1.9$ procedures. Regarding the latter anti-VEGF injection before $\mathrm{V}_{0}$, bevacizumab was the drug employed in 106 out of 112 eyes, while ranibizumab and aflibercept were the latter drugs injected in 4 and 2 eyes, respectively.

Mean \pm SD time interval between following visits was $110.7 \pm 37.5$ days within $\mathrm{V}_{0}$ and $\mathrm{V}_{-1}$ and $80.8 \pm 39.7$ days within $\mathrm{V}_{-1}$ and $\mathrm{V}_{-2}$, respectively $(P<0.0001)$ (Fig. 1). The average difference between these two intervals was $29.9 \pm$ 48.5 days.

\section{Functional and anatomic outcomes}

Mean \pm SD BCVA was statistically worse at the inclusion $\left(\mathrm{V}_{0}\right)$ visit as compared with the immediately preceding $\left(\mathrm{V}_{-1}\right)$ visit $(0.50 \pm 0.43 \operatorname{LogMAR}$ and $0.45 \pm 0.38$ LogMAR at the $\mathrm{V}_{0}$ and $\mathrm{V}_{-1}$ visits, respectively; $P=$ 0.046). No differences in BCVA were detected between the $\mathrm{V}_{-1}$ and $\mathrm{V}_{-2}$ visits $\left(0.45 \pm 0.37 \operatorname{LogMAR}\right.$ at the $\mathrm{V}_{-2}$ visit; $P=0.834$ ) (Table 2, Fig. 2). Change in BCVA was $-8.8 \pm 29.3 \%$ in eyes who required at least 5 injections in the previous year (29 eyes) and $-2.0 \pm 29.3 \%$ in

Table 1 Characteristics of patients included in the analysis

\begin{tabular}{lc} 
Number of eyes enrolled (patients) & $112(100)$ \\
Age (years), mean (SD) & $79.1(7.6)$ \\
Gender, $n(\%)$ & \\
M & $51(51.0 \%)$ \\
F & $49(49.0 \%)$ \\
Previous anti-VEGF Injections, mean (SD) & $15.0(10.1)$ \\
\hline
\end{tabular}

$S D$ standard deviation, $n$ number of eyes, $B C V A$ best-corrected visual acuity (logMAR [logarithm of the minimum angle of resolution]), $V E G F$ vascular endothelial growth factor 
Fig. 1 Graph showing the time interval between following visits. The COVID-19 pandemic caused a significant delay in the assessment of patients with neovascular AMD. The time interval between $\mathrm{V}_{0}$ (the inclusion visit during the COVID-19 pandemic) and the immediately preceding visit $\left(\mathrm{V}_{-1}\right)$ was significantly longer than the interval between $\mathrm{V}_{-1}$ and the preceding visit $\left(\mathrm{V}_{-2}\right)$. The inclusion visit was performed during the COVID-19 pandemic (from March 9, 2020, through June 12, 2020) and this period is highlighted in red in this graph

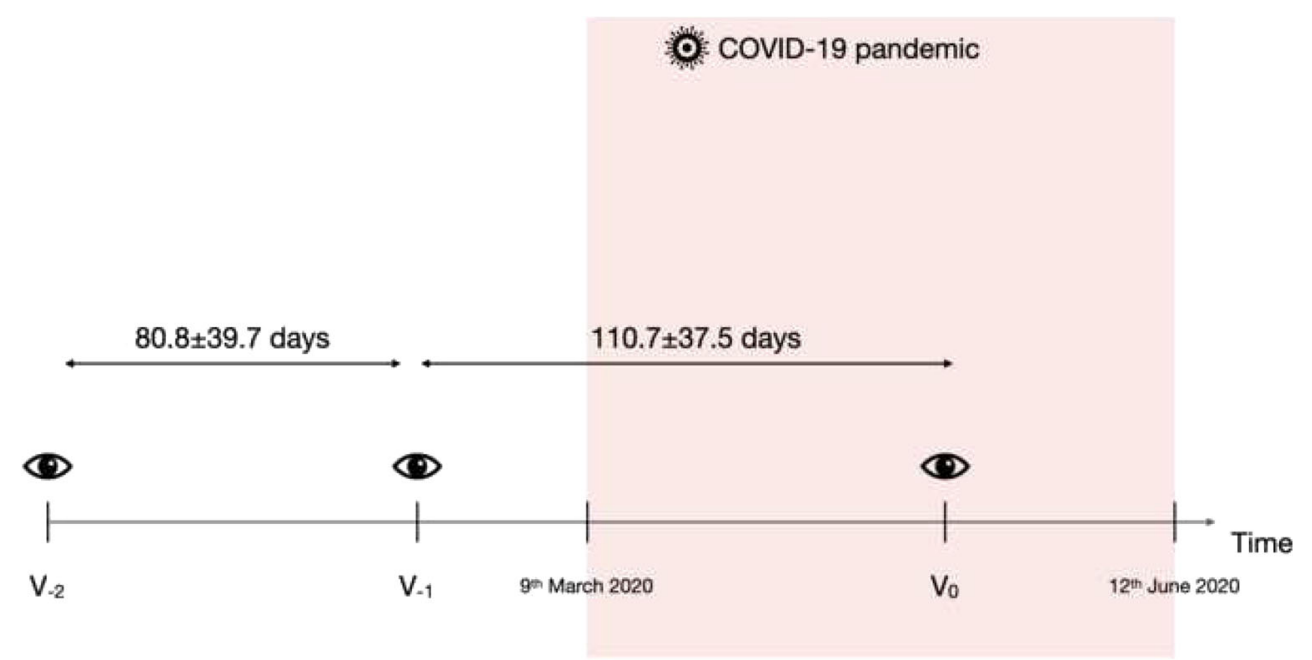

patients who required less than 5 injections in the last year (83 eyes). The latter analysis suggested that eyes requiring more injections had a worse reduction in visual acuity at $\mathrm{V}_{0}$.

On structural OCT, 91 out of $112(81.2 \%)$ neovascular AMD eyes displayed the evidence of MNV exudation at the $\mathrm{V}_{0}$ visit, while $77(68.7 \%)$ and $85(75.9 \%)$ eyes exhibited signs of MNV exudation at the $\mathrm{V}_{-1}\left(P=0.022\right.$ vs $\left.\mathrm{V}_{0}\right)$ and $\mathrm{V}_{-2}\left(P=0.148\right.$ vs $\left.\mathrm{V}_{-1}\right)$ visits, respectively (Table 2, Figs. 3 and 4).

\section{Regression analysis}

Results of multiple regression analysis are summarized in Table 3. In multiple regression analysis, the difference in BCVA between $\mathrm{V}_{0}$ and $\mathrm{V}_{-1}$ visits was significantly associated with the interval time within these two visits $(P=0.026)$.

\section{Discussion}

In this report we estimated the impact of delayed care on the visual and anatomic outcomes of patients with neovascular AMD. Overall, we demonstrated that the COVID-19 pandemic significantly delayed the in-person visits and subsequent possible intravitreal procedures. More importantly, we proved that this deferral was significantly associated with worse short-term outcomes in these patients.

In a previous report, [24] our group demonstrated a significant reduction in average age among patients undergoing visits and intravitreal procedures during the 2020 COVID-19 quarantine, as compared with the same period in 2019 . Furthermore, among patients referring to a medical retina practice who presented during the COVID-19 period, the prevalence of AMD was lower than among those who presented during the pre-COVID-19 period. Based on these findings, we concluded that outpatient care in AMD patients was
Table 2 Functional and anatomic parameters at different visits

\begin{tabular}{llll}
\hline & $\mathrm{V}_{-2}$ & $\mathrm{~V}_{-1}$ & $\mathrm{~V}_{0}$ \\
\hline BCVA (LogMAR), mean (SD) & $0.45(0.37)$ & $0.45(0.38)$ & $0.50(0.43)$ \\
OCT evidence of MNV exudation, $n(\%)$ & $85(75.9)$ & $0.834^{\mathrm{b}}$ & $0.046^{\mathrm{a}}$ \\
& & $77(68.7)$ & $91(81.2)$ \\
OCT evidence of intraretinal fluid, $n(\%)$ & $50(44.6)$ & $0.148^{\mathrm{b}}$ & $0.022^{\mathrm{a}}$ \\
OCT evidence of subretinal fluid, $n(\%)$ & & $45(40.2)$ & $50(44.6)$ \\
& $51(45.5)$ & $0.294^{\mathrm{b}}$ & $0.294^{\mathrm{a}}$ \\
OCT evidence of SHRM, $n(\%)$ & & $42(37.5)$ & $56(50.0)$ \\
& $46(41.1)$ & $0.139^{\mathrm{b}}$ & $0.040^{\mathrm{a}}$ \\
& & $37(33.0)$ & $40(35.7)$ \\
\hline
\end{tabular}

$B C V A$ best-corrected visual acuity (logMAR (logarithm of the minimum angle of resolution)), $S D$ standard deviation, $O C T$ optical coherence tomography, $M N V$ macular neovascularization, $n$ number of eyes, SHRM subretinal hyperreflective material

${ }^{\mathrm{a}}$ Comparison versus $\mathrm{V}_{-1} ;{ }^{\mathrm{b}}$ comparison versus $\mathrm{V}_{-2}$ 


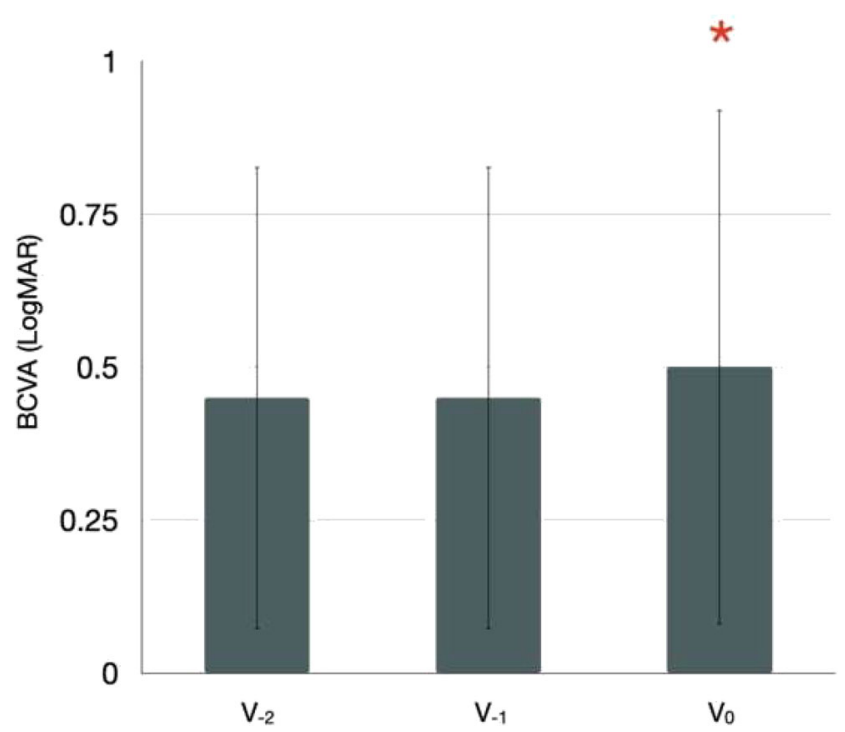

Fig. 2 Bar chart showing differences in visual acuity between visits. Error-bar chart displaying study cohort's visual acuity at different visits. The height of the bars corresponds to the mean value, while the error bars represent the standard deviation (SD) of the data. Best-corrected visual acuity (BCVA) was statistically worse at the inclusion $\left(\mathrm{V}_{0}\right)$ visit as compared with the immediately preceding $\left(\mathrm{V}_{-1}\right)$ visit. Conversely, no differences were detected in terms of BCVA between the $\mathrm{V}_{-1}$ and immediately preceding $\mathrm{V}_{-2}$ visits. Red asterisks indicate significative differences in the comparison with the previous visit

most significantly impacted by the COVID-19 pandemic in a referral medical retina practice.

This shrinking in outpatient volume may be related to different factors. First, ophthalmology teams have adopted new ways of working to minimize risk to patients and staff at the same time as optimizing treatment and care of ocular disorders. In details, ophthalmology departments conceived riskstratification guidelines to decide if patients needed to be visit in-person or could be deferred [19, 20, 23]. Such guidelines were also consequent to shortages of personal protective equipment (PPE) and absence of well-established protocols that might reduce personnel risk. Also, COVID-19 infections among health workers have been dramatically contracting the numbers of available staff. Second, patients were also limited in travelling between regions and this may have limited their capability to attend visits and intravitreal treatments. Third, many patients also postponed visits as fears of exposure to COVID-19 are causing people to avoid hospital help. Accordingly, results of the present study demonstrated a significant increase in the time interval between following visits during this real-life emergency setting.

As discussed above, fixed-interval regimens are characterized by a significant treatment burden for patients, caregivers and physicians [33]. Assuming this, fixed-interval regimens are often unfeasible in clinical practice and less frequent dosing protocols (i.e. PRN and TREX) have been widely employed in patients with neovascular AMD [7, 33]. However, these non-continuous protocols require an adequate number of ocular examinations in order to avoid undertreatment in these patients. Holz and colleagues [33] reviewed 2227 patients with neovascular AMD and receiving anti-VEGF treatment in clinical practice. The latter study of real-life anti-VEGF therapy demonstrated that more frequent visits and injections were associated with greater improvements in visual outcome.

We add to the literature by reporting the impact of delayed care secondary to the COVID-19 pandemic on the short-term visual and anatomic outcomes of patients with neovascular AMD under a PRN approach. Importantly, we proved that this delay was significantly associated with worse functional and anatomical short-term outcomes. In details, at the inclusion visit, which was performed during or immediately after the Italian COVID-19 quarantine, patients had worse visual acuities as compared with the previous visits. Similarly, at the same visit, a larger portion of patients had OCT evidences of exudative disease activity $\left(81.2 \%\right.$ at the $\mathrm{V}_{0}$ and $68.7 \%$ at the $\mathrm{V}_{-1}$ ). Importantly, no similar differences were detected

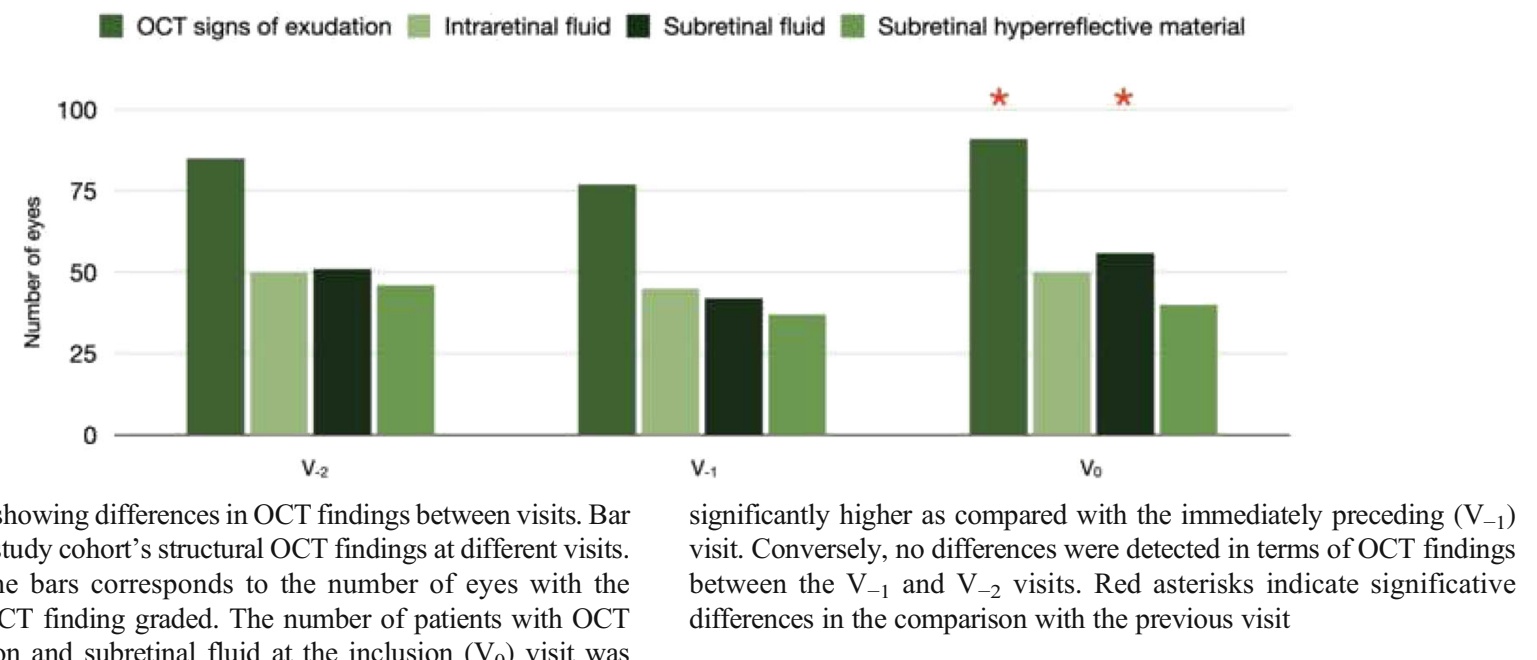

Fig. 3 Bar chart showing differences in OCT findings between visits. Ba chart displaying study cohort's structural OCT findings at different visits. The height of the bars corresponds to the number of eyes with the corresponding OCT finding graded. The number of patients with OCT sings of exudation and subretinal fluid at the inclusion $\left(\mathrm{V}_{0}\right)$ visit was 
Fig. 4 Structural OCT from a patient with exudative neovascular AMD. Top panel, $\mathrm{V}_{-2}$ visit; middle panel, $\mathrm{V}_{-1}$ visit; bottom panel, $\mathrm{V}_{0}$ (inclusion) visit. The green arrows on the nearinfrared reflectance images (left) shows the location and direction of the structural optical coherence tomography (OCT) B-scans (right images). At the $\mathrm{V}_{-2}$ and $\mathrm{V}_{-1}$ visits, the structural OCT B-scans show the presence of a fibrovascular pigment epithelial detachment associated with a small amount of subretinal hyperreflective material (SHRM). At the inclusion visit performed during the COVID-19 pandemic $\left(\mathrm{V}_{0}\right)$, the structural OCT B-scan displays an increased quantity of SHRM with associated subretinal fluid. Similarly, the best-corrected visual acuity (BCVA) was significantly worse at the $\mathrm{V}_{0}$ visit, as compared with both the preceding visits $\left(\mathrm{V}_{-1}\right.$ and $\left.\mathrm{V}_{-2}\right)$. The time interval between following visits was 63 (within $\mathrm{V}_{-1}$ and $\mathrm{V}_{-2}$ ) and 147 (within $\mathrm{V}_{0}$ and $\mathrm{V}_{-1}$ ) days
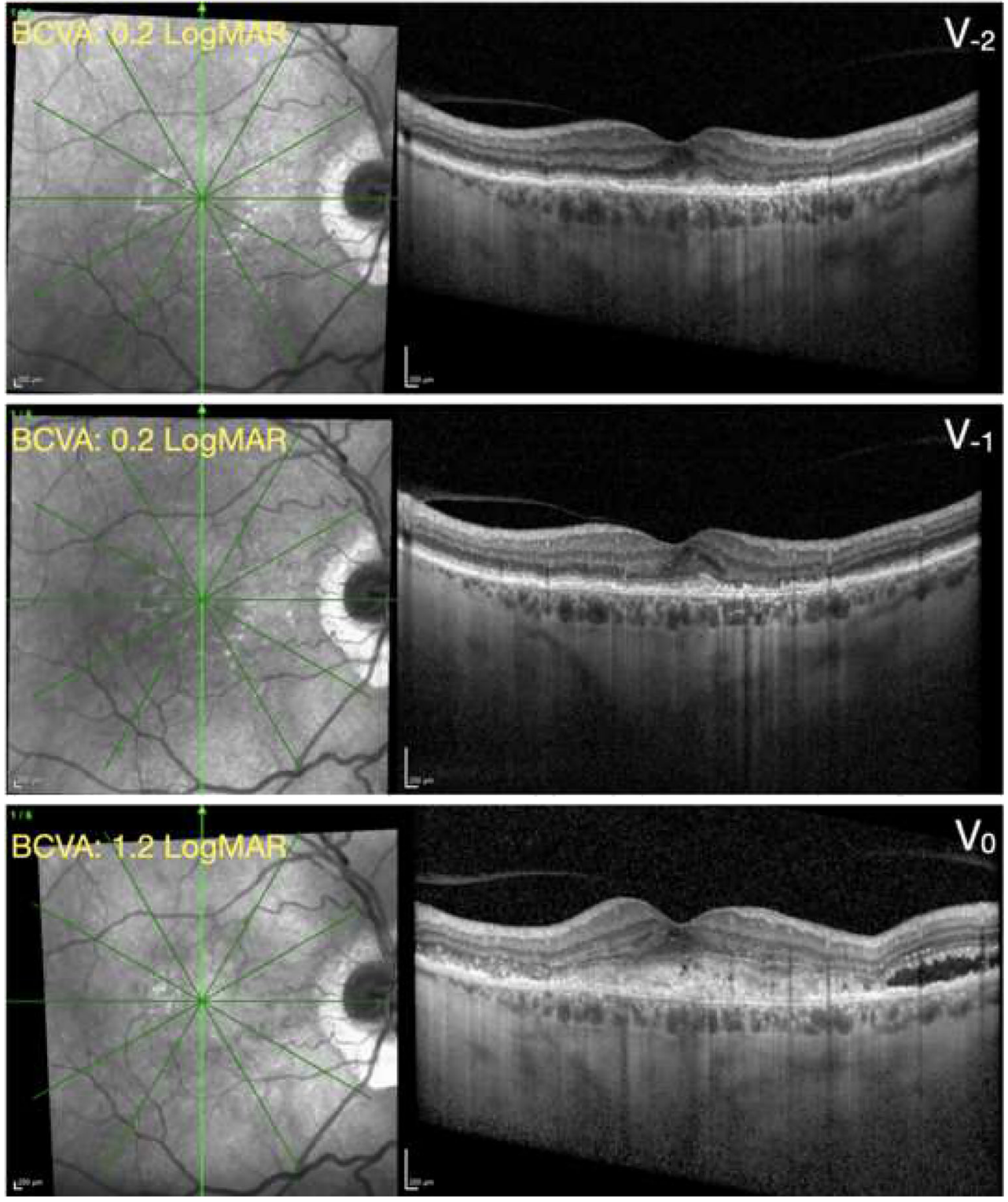

between the $\mathrm{V}_{-1}$ and $\mathrm{V}_{-2}$ visits, which were both performed before the COVID-19 pandemic.

The finding in our study of a worse visual acuity at the visit performed during a real-life emergency setting may indicate that a longer time interval between visits may be causing a reduction in visual acuity in these patients. Indeed, the present study highlights the distinctive relationship between drop in visual acuity and time interval between visits: considering
Table 3 Results of multiple regression analysis of the association between change in visual acuity between $\mathrm{V}_{0}$ and $\mathrm{V}_{-1}$ and other variables

\begin{tabular}{lcc}
\hline & Standardized B coefficient (SE) & $P$ value \\
\hline Time interval between $\mathrm{V}_{0}$ and $\mathrm{V}_{-1}$ & 0.267 & 0.026 \\
Number of anti-VEGF injections in the last year before $\mathrm{V}_{0}$ & 0.082 & 0.404 \\
Number of previous anti-VEGF injections & -0.037 & 0.694 \\
BCVA at $\mathrm{V}_{-1}$ & 0.024 & 0.799 \\
Age & 0.189 & 0.053 \\
Gender & 0.096 & 0.316 \\
\hline
\end{tabular}

$V E G F$ vascular endothelial growth factor, $B C V A$ best-corrected visual acuity 
change in visual acuity within visits as dependent variable, we observed a direct relationship between these two variables, even after accounting for confounding factors, such as age and gender.

Although the COVID-19 pandemic is limited in time, this could last about 2 years. In addition, our findings might be applied to other emergency settings. Moreover, our results highlight the relevance of efforts to not delay in-person visits and subsequent intravitreal procedures. As an example, we have recently proposed a novel discontinuous regimen, which was termed "Triple and Plan (TriPla)" (Querques G. Presented during a webinar ESASO in 2020), that is aimed at reducing the number of in-person visits by performing a complete ophthalmologic evaluation every three injections. Furthermore, administrators have been relocating ophthalmology teams and services away from departments caring for patients with COVID-19. This may reduce patients' and healthcare workers' fears of exposure to COVID-19.

Limitations of this study include the analysis of a single community setting in northern Italy, which to date is one of the most affected regions worldwide. The heterogenicity of our study cohort may be considered as another limitation. In details, patients had a large range in visual acuity and number of intravitreal injections before $\mathrm{V}_{0}$. However, the aim of this study was to provide a rough estimate of the COVID-19 impact on patients with neovascular AMD in a tertiary referral centre. Of note, we performed a multiple regression analysis including main patients' clinical characteristics. Since multiple linear regression analysis allows us to estimate the association between a given dependent variable and other parameters, it provides a way of adjusting for (or accounting for) potentially confounding variables (i.e. study cohort variability). Finally, we did not provide a quantification of OCT signs of exudation. Assuming that presence of some SRF was demonstrated to be tolerable in patients with neovascular AMD [34], this represents a further limitation in our study. The strengths of our study include the consecutive design and the large sample size of consecutively enrolled patients.

In conclusion, in a tertiary referral retina unit, the COVID19 pandemic resulted in a significant delay in neovascular AMD patient care. Importantly, this postponement proved to be significantly associated with worse short-term outcomes in these patients. These findings may help broaden our knowledge regarding the natural history of AMD and the management of these patients in an emergency setting. Future studies with extended longitudinal follow-up of this cohort may provide additional substantive information. Importantly, these studies may clarify whether the COVID-19 pandemic may have a negative impact on long-term outcomes. Finally, our findings will help ophthalmologists to evaluate the risk of vision loss associated with extending interval between visits and should be taken together with other factors including prevalence of COVID-19 within the community and individual patient's systemic comorbidities.

Authors' contributions EB: Study supervision. Concept and study design. Data collection. Data interpretation. Data analysis and statistics. Drafting, revision and final approval of manuscript.

DG: Concept and study design. Data collection. Data interpretation. Data analysis and statistics. Drafting, revision and final approval of manuscript.

GV: Concept and study design. Data collection. Data interpretation. Data analysis and statistics. Drafting, revision and final approval of manuscript.

RS: Data collection. Revision and final approval of manuscript.

MB: Data collection. Revision and final approval of manuscript.

LQ: Data collection. Revision and final approval of manuscript.

IZ: Data collection. Revision and final approval of manuscript.

FP: Data collection. Revision and final approval of manuscript.

FB: Study supervision. Concept and study design. Data interpretation. Revision and final approval of manuscript.

GQ: Study supervision. Concept and study design. Data interpretation. Revision and final approval of manuscript.

Data availability The data used to support the findings of this study are available from the corresponding author upon request.

\section{Compliance with ethical standards}

Conflict of interest Francesco Bandello is a consultant for Alcon (Fort Worth, Texas, USA), Alimera Sciences (Alpharetta, Georgia, USA), Allergan Inc. (Irvine, California, USA), Farmila-Thea (ClermontFerrand, France), Bayer Shering-Pharma (Berlin, Germany), Bausch And Lomb (Rochester, New York, USA), Genentech (San Francisco, California, USA), Hoffmann-La-Roche (Basel, Switzerland), Novagali Pharma (Évry, France), Novartis (Basel, Switzerland), Sanofi-Aventis (Paris, France), Thrombogenics (Heverlee, Belgium), Zeiss (Dublin, USA). Giuseppe Querques is a consultant for Alimera Sciences (Alpharetta, Georgia, USA), Allergan Inc. (Irvine, California, USA), Amgen (Thousand Oaks, USA), Bayer Shering-Pharma (Berlin, Germany), Heidelberg (Germany), KBH (Chengdu; China), LEH Pharma (London, UK), Lumithera (Poulsbo; USA), Novartis (Basel, Switzerland), Sandoz (Berlin, Germany), Sifi (Catania, Italy), SooftFidea (Abano, Italy), Zeiss (Dublin, USA). The other authors have no disclosures.

Ethical approval This article does not contain any studies with animals performed by any of the authors. All procedures performed in studies involving human participants were in accordance with the ethical standards of the (place name of institution and/or national research committee) and with the 1964 Helsinki declaration and its later amendments or comparable ethical standards.

Informed consent Informed consent was obtained from all individual participants included in the study.

\section{References}

1. Jager RD, Mieler WF, Miller JW (2008) Age-related macular degeneration. N Engl J Med 358:2606-2617. https://doi.org/10.1056/ NEJMra0801537

2. Spaide RF, Jaffe GJ, Sarraf D, et al (2019) Consensus nomenclature for reporting neovascular age-related macular degeneration Data: 
Consensus on Neovascular Age-Related Macular Degeneration Nomenclature Study Group. Ophthalmology. https://doi.org/10. 1016/j.ophtha.2019.11.004

3. Borrelli E, Sarraf D, Freund KB, Sadda SR (2018) OCT angiography and evaluation of the choroid and choroidal vascular disorders. Prog Retin Eye Res. https://doi.org/10.1016/j.preteyeres.2018.07. 002

4. Querques L, Parravano M, Borrelli E et al (2020) Anatomical and functional changes in neovascular AMD in remission: comparison of fibrocellular and fibrovascular phenotypes. $\mathrm{Br} \mathrm{J}$ Ophthalmol. https://doi.org/10.1136/bjophthalmol-2018-313685

5. Martin DF (2018) Evolution of Intravitreal therapy for retinal diseases - from CMV to CNV: the LXXIV Edward Jackson memorial lecture. Am J Ophthalmol. https://doi.org/10.1016/j.ajo. 2017.12.019

6. Keane PA, Sadda SR (2012) Development of anti-VEGF therapies for intraocular use: a guide for clinicians. J Ophthalmol. https://doi. org/10.1155/2012/483034

7. Solomon SD, Lindsley K, Vedula SS et al (2014) Anti-vascular endothelial growth factor for neovascular age-related macular degeneration. Cochrane Database Syst Rev. https://oi.org/10.1002/ 14651858.CD005139.pub3

8. Brown DM, Kaiser PK, Michels M et al (2006) Ranibizumab versus verteporfin for neovascular age-related macular degeneration. $\mathrm{N}$ Engl J Med. https://doi.org/10.1056/NEJMoa062655

9. Rosenfeld PJ, Brown DM, Heier JS et al (2006) Ranibizumab for neovascular age-related macular degeneration. N Engl J Med. https://doi.org/10.1056/NEJMoa054481

10. Regillo CD, Brown DM, Abraham P et al (2008) Randomized, double-masked, sham-controlled trial of ranibizumab for neovascular age-related macular degeneration: PIER study year 1 . Am J Ophthalmol. https://doi.org/10.1016/j.ajo.2007.10.004

11. Sarwar S, Clearfield E, Soliman MK et al (2016) Aflibercept for neovascular age-related macular degeneration. Cochrane Database Syst Rev. https://doi.org/10.1002/14651858.CD011346.pub2

12. Heier JS, Brown DM, Chong V et al (2012) Intravitreal aflibercept (VEGF trap-eye) in wet age-related macular degeneration. Ophthalmology. https://doi.org/10.1016/j.ophtha.2012.09.006

13. Wecker T, Grundel B, Reichl S et al (2019) Anti-VEGF injection frequency correlates with visual acuity outcomes in pro re nata neovascular AMD treatment. Sci Rep. https://doi.org/10.1038/ s41598-019-38934-8

14. Freund KB, Korobelnik J-F, Devenyi R, et al (2015) TREATAND-EXTEND REGIMENS WITH ANTI-VEGF AGENTS IN RETINAL DISEASES: A Literature Review and Consensus Recommendations. Retina. https://doi.org/10.1097/IAE. 0000000000000627

15. Gupta OP, Shienbaum G, Patel AH et al (2010) A treat and extend regimen using ranibizumab for neovascular age-related macular degeneration: clinical and economic impact. Ophthalmology. https://doi.org/10.1016/j.ophtha.2010.02.032

16. Shienbaum G, Gupta OP, Fecarotta C et al (2012) Bevacizumab for neovascular age-related macular degeneration using a treat-andextend regimen: clinical and economic impact. Am J Ophthalmol. https://doi.org/10.1016/j.ajo.2011.08.011

17. Silva R, Berta A, Larsen M, et al (2018) Treat-and-extend versus monthly regimen in neovascular age-related macular Degeneration: Results with Ranibizumab from the TREND Study. Ophthalmology. https://doi.org/10.1016/j.ophtha.2017.07.014

18. Berg K, Hadzalic E, Gjertsen I, et al (2016) Ranibizumab or bevacizumab for neovascular age-related macular degeneration according to the Lucentis compared to Avastin study treat-and-extend Protocol: Two-Year Results. Ophthalmology. https://doi.org/10. 1016/j.ophtha.2015.09.018
19. Parravano M, Borrelli E, Costanzo E et al (2020) Protect healthcare workers and patients from COVID-19: the experience of two tertiary ophthalmology care referral centers in Italy. Ophthalmol Therapy. https://doi.org/10.1007/s40123-020-00251-z

20. Borrelli E, Sacconi R, Querques L et al (2020) Taking the right measures to control COVID-19 in ophthalmology: the experience of a tertiary eye care referral center in Italy. Eye. https://doi.org/10. 1038/s41433-020-0880-6

21. Corradetti G, Corvi F, Nguyen TV, Sadda SR (2020) Management of neovascular age-related macular degeneration during the COVID-19 pandemia. Ophthalmol Retin. https://doi.org/10.1016/ j.oret.2020.05.015

22. Shmueli O, Chowers I, Levy J (2020) Current safety preferences for intravitreal injection during COVID-19 pandemic. Eye (Lond). https://doi.org/10.1038/s41433-020-0925-x

23. Iovino C, Caporossi T, Peiretti E (2020) Vitreoretinal surgery tip and tricks in the era of COVID-19. Graefes Arch Clin Exp Ophthalmol 1-2 . https://doi.org/10.1007/s00417-020-04800-x

24. Borrelli E, Grosso D, Vella G et al (2020) Impact of COVID-19 on outpatient visits and intravitreal treatments in a referral retina unit: let's be ready for a plausible "rebound effect". Graefes Arch Clin Exp Ophthalmol. https://doi.org/10.1007/s00417-020-04858-7

25. Holz FG, Tadayoni R, Beatty S et al (2016) Key drivers of visual acuity gains in neovascular age-related macular degeneration in real life: findings from the AURA study. Br J Ophthalmol. https://doi. org/10.1136/bjophthalmol-2015-308166

26. Holladay JT (1997) Proper method for calculating average visual acuity. J Refract Surg 13:388-391

27. Huang Y, Gangaputra S, Lee KE et al (2012) Signal quality assessment of retinal optical coherence tomography images. Invest Ophthalmol Vis Sci 53:2133-2141. https://doi.org/10.1167/iovs. 11-8755

28. Sadda SR, Guymer R, Holz FGFG et al (2018) Consensus definition for atrophy associated with age-related macular degeneration on OCT: classification of atrophy report 3. Ophthalmology. https:// doi.org/10.1016/j.ophtha.2017.09.028

29. Lee SY, Stetson PF, Ruiz-Garcia H, et al (2012) Automated characterization of pigment epithelial detachment by optical coherence tomography Investig Ophthalmol Vis Sci 53. https://doi.org/10. 1167/iovs.11-8188

30. Jung JJ, Chen CY, Mrejen S et al (2014) The incidence of neovascular subtypes in newly diagnosed neovascular age-related macular degeneration. Am J Ophthalmol 158:769-779.e2. https:// doi.org/10.1016/j.ajo.2014.07.006

31. Dansingani KK, Tan ACS, Gilani F et al (2016) Subretinal hyperreflective material imaged with optical coherence tomography angiography. Am J Ophthalmol 169:235-248. https://doi.org/ 10.1016/j.ajo.2016.06.031

32. Nassisi M, Lei J, Abdelfattah NS et al (2019) OCT risk factors for development of late age-related macular degeneration in the fellow eyes of patients enrolled in the HARBOR study. Ophthalmology. https://doi.org/10.1016/j.ophtha.2019.05.016

33. Holz FG, Tadayoni R, Beatty S et al (2015) Multi-country real-life experience of anti-vascular endothelial growth factor therapy for wet age-related macular degeneration. Br J Ophthalmol. https:// doi.org/10.1136/bjophthalmol-2014-305327

34. Guymer RH, Markey CM, McAllister IL et al (2019) Tolerating subretinal fluid in neovascular age-related macular degeneration treated with ranibizumab using a treat-and-extend regimen: FLUID study 24-month results. Ophthalmology. https://oi.org/ 10.1016/j.ophtha.2018.11.025

Publisher's note Springer Nature remains neutral with regard to jurisdictional claims in published maps and institutional affiliations. 